\title{
OBSERVATIONS SUR LES BLASTOCYSTIS
}

\author{
Par G. LAVIER
}

Les Blastocystis, ces parasites unicellulaires très banaux de l'intestin chez un grand nombre d'hôtes, hommes ou animaux (mammifères, oiseaux, reptiles, amphibiens, insectes et annélides mêmes), sont connus depuis longtemps et ont déjà donné lieu à nombre d'études. Cependant, leur statut exact est loin d'être éclairci. Des observations faites au cours de plusieurs années me permettent, sinon de jeter sur ce sujet une pleine lumière, du moins de fournir quelques précisions.

Elles ont été réalisées sur des Blastocystis de provenances suivantes : homme, souris blanche, amphibiens (Rana esculenta, R. temporaria, Bufo bufo), sangsue (Hæmopis sanguisuga). L'étude morphologique de ces parasites a porté sur les formes observées directement dans les déjections ou le contenu intestinal des hôtes ou sur celles dévelcppées dans les cultures qui en provenaient. La culture des Blastocystis est, on le sait depuis Barrett (1921), facile à obtenir dans un milieu organique riche en protides, comme le sérum, dilué en liquide isotonique. En fait, le liquide de Ringer additionné de 5 à 10 p. 100 de sérum de cheval, ou autre, constitue pour cela un excellent milieu. Mais le sérum n'est pas nécessaire et le milieu ovo-mucoïde de Boeck (un blane d'œuf battu stérilement dans un litre de liquide de Ringer) est également favorable quoique donnant une luxuriance bien moindre. Avec les parasites provenant de poïkilothermes la culture, quel que soit le milieu, s'obtient sans difficulté à la température du laboratoire : non seulement elle ne se réalise pas à $37^{\circ}$, mais si l'on place à cette température une culture déjà en train, celle-ci est rapidement arrêtée et les organismes tués. Inversement, les blastocystes d'animaux à sang chaud ne poussent qu'à une température de $35^{\circ}$ à $37^{\circ}$ et l'on n'obtient au-dessous qu'une multiplication très faible et transitoire. $\mathrm{Si}$, pour la richesse des cultures, le milieu ovo-mucoïde est certainement inférieur au milieu dilué, il a du moins l'avantage, étant plus pauvre, de fournir des microorganismes à cytoplasme moins chargé et ainsi d'observation cyto-

Ans. de Parastrologie, T. XXVII, N $^{\circ} 1-2-3 .-1952$. 
logique plus aisée ; certains aspects y apparaissent aussi que l'on n'observe pas dans l'autre. Dans les deux milieux, la culture se réalise bien en aérobiose, mais si l'on ajoute une légère couche d'huile de paraffine, la poussée est plus forte et prolongée ; les blastocystes s'accommodent donc bien d'une anaérobiose au moins relative. Les parasites d'homéothermes paraissent plus exigeants, au point de vue nutritif, que ceux d'animaux à sang froid, et il est difficile avec eux d'avoir des cultures d'une certaine durée en ovomucoïde. Mais avec ce dernier milieu, les Blastocystis d'amphibiens et de sangsue fournissent un excellent matériel d'étude (1).

Etant donné l'abondance de la littérature sur la morphologie des Blastocystis, je ne la nésumerai pas ici, me contentant de signaler à l'occasion ce en quoi je suis d'accord ou en contradiction avec les auteurs antérieurs.

Les Blastocystis se présentent sous deux aspects principaux correspondant à deux phases : une phase végétative avec mode de multiplication très simple ; une phase de sporulation, mode de multiplication très spécial qui transforme brusquement l'organisme entier en un certain nombre d'éléments de résistance, des spores.

\section{Phase végétative}

L'aspect sphéroïde des Blastocystis dans les déjections ou le contenu intestinal prélevé à l'autopsie est trop connu pour qu'il soit utile de s'étendre à ce sujet. Il est toutefois bon d'insister sur un point : c'est qu'une proportion parfois notable des individus que l'on observe dans ces conditions sont morts, ainsi que le démontre facilement l'épreuve des colorants. Il convient donc d'être très prudent dans l'interprétation et de ne pas se laisser séduire, ce qui est arrivé à plusieurs, par certains aspects particulièrement favorables à des inductions faciles et qui ne correspondent qu'à des altérations cadavériques. En fait, la morphologie générale du parasite vivant varie assez peu. Dans certaines infections considérables d'amphibiens, le nombre de parasites peut être tel qu'ils obstruent la lumière intestinale d'un véritable faux-tissu d'éléments devenus polyédriques par le tassement ; le nombre des cellules mortes est alors considérable. Le diamètre des sphéroïdes, mème pour une souche unique, varie dans de très larges proportions : de 1 à 6 ; j'ai même vu, chez un Bufo bufo, une variation de 1 à 8 . La taille ne

(1) J'ai utilisé aussi les autres milieux suivants : sérum coagulé surmonté de liquide de Ringer : résultats intermédiaires entre le sérum dilué et l'ovomucoîde, pullulation bactérienne trop rapide ; Ringer-peptone : résultats nuls ; eau de levures : résultats nuls; cau de pomme de terre : poussée faible. 
saurait done guère être utilisée comme moyen de distinction. Il est à noter cependant que, dans l'ensemble, le diamètre moyen paraît plus grand chez les animaux à sang froid.

Cet élément sphéroïde (kyste primaire d'Alexeieff, kystoïde de Chatton) représente la forme végétative du parasite ; il est, dans son ensemble, constitué de trois parties : le cytoplasme, la vacuole centrale, la membrane externe.

$1^{\circ}$ Le cytoplasme. - La sphère constituant la grosse vacuole centrale et qui est légèrement excentrique donne au cytoplasme un aspect, en coupe optique, qui est annulaire ou plus souvent encore en croissant. A frais, il est finement granuleux, avec des inclusions dispersées de réfringence différente; l'une, relativement grande, de réfringence assez faible, est le noyau dont la structure n'apparaît qu'après coloration ; l'hématoxyline ferrique la révèle comme absolument caractéristique, telle qu'elle était déjà décrite en 1910 par Alexeieff, revue par Beaurepaire-Aragão (1922), Grassé (1926), noyau sphérique ou ovalaire, entouré d'une mince membrane et présentant à un pôle une épaisse calotte chromatique et, dans le reste, un espace parfois clair mais le plus souvent empli d'une fine poussière chromatique.

Quant aux petites inclusions, elles sont de nature diverse. Les unes très réfringentes appartiennent au chondriome. Pour bien observer celui-ci, il faut s'adresser aux cultures ovo-mucoïdes qui donnent des individus à cytoplasme moins chargé que le milieu au sérum et ainsi de lecture bien plus facile. Le vert janus colore les mitochondries de façon nette mais les altère très rapidement en sphérules ; il en est de même pour le violet dalhịia qui les teinte en lilas mais les déforme presque aussitôt ; c'est la solution iodo-iodurée forte (à 4 p. 100 d'iode), suivant la technique de Guilliermond qui en permet l'observation la plus facile et la plus précise : les mitochondries se présentent alors sous l'aspect de bâtonnets plus ou moins longs et souvent recourbés, dont certains offrent un aspect de cavulation (fig. 1). Ces éléments sont dispersés dans le cytoplasme, y occupant une situation relativement superficielle, et ils doivent probablement jouer un rôle important dans la constitution de la membrane sporale au moment de la sporulation. Alexeieff (1910) avait signalé, dans le

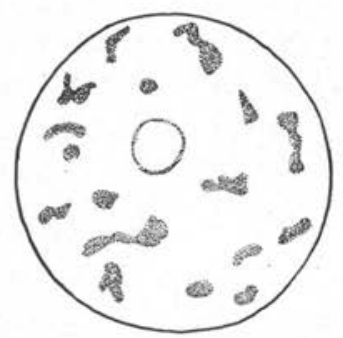

FIG. 1. - Chondricme de Blastocystis (Rana esculenta ; culture en liquide ovo-mucoïde ; solution iodo-iodurée forte). 
cytoplasme des blastocystes colorés par l'hématoxyline ferrique, des bâtonnets et des granules sidérophiles et les avait interprétés comme des mitochondries. Grassé leur a contesté cette nature, étant donné la fixation utilisée. Il n'y a pas de doute cependant qu'il s'agit bien d'éléments du chondriome mais très réduits et très altérés par une technique impropre. On ne saurait parler en tout cas, comme cela a été dit en faveur d'une interprétation d'affinité, de pauvreté mitochondriale des Blastocystis, si l'on tient compte du faible volume que représente le cytoplasme actif.

Mais il y a des granulations réfringentes d'autre nature : le soudan III colore un nombre toujours très faible de petites sphérules

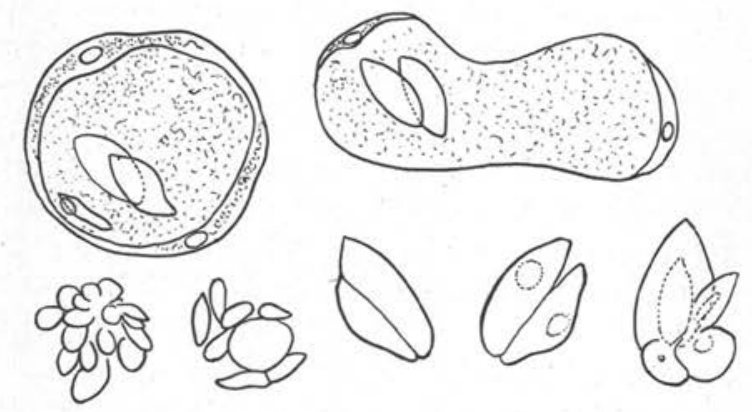

FIc. 2. - Formations cristalloïdes dans la vacuole centrale. Rangée supérieure : deux individus dont un en division; rangée inférieure : amas cristalloïdes observès dans les vacuoles d'autres individus (formes intestinales; Rana esculenta 200 ; à frais).

lipidiques ( 2 ou 3 pour les cultures en ovo-mucoïde, un peu plus volumineux pour le Ringer-sérum). Le bleu de crésyl brillant ne met en évidence aucune enclave métachromatique et on ne voit pas sur quelle base Alexeieff a pu parler de «paravolutine » et de «métavolutine ». On n'observe que très rarement du glycogène et toujours en inclusions extrêmement réduites ; il n'en sera plus de même au moment de la sporulation, où le glycogène est par contre toujours très abondant.

$2^{\circ}$ La vacuole centrale. - Le contenu de la grosse vacuole centrale forme une sphère excentrique contenue dans la sphère totale. Elle a reçu divers noms : Innenkörper, corps interne, corps central, corps de réserve, etc... C'est en réalité une vacuole comme il en existe dans nombre de protistes.

L'aspect à frais de son contenu est variable. Dans la majorité des cas il est homogène, plus ou moins finement spumeux; sa réfrin- 
gence aussi est variable ; elle est parfois très forte et alors cette formation qui est généralement incolore prend une légère teinte jaune-verdâtre. Si l'on provoque, par pression, l'éclatement d'un élément, on voit le contenu s'écouler lentement ; il s'agit donc d'un liquide de forte viscosité. Parfois ce liquide est dissocié en deux phases dont l'une, interne, est manifestement plus compacte ; elle peut même prendre un aspect solide avec apparence de formations arrondies ou de cristalloïdes isolés ou maclés (fig. 2), ou même de figures très complexes ; Alexeieff en a décrit cinq types différents d'aspects, sans compter les atypiques! De telles formes ne s'observent d'ailleurs pas dans les cultures. Mais il est un aspect que l'on peut rencontrer dans les blastocystes de mammifères, aussi bien dans les fèces que dans les cultures en Ringer-sérum : c'est la vacuole entièrement remplie de sphérules réfringentes, parfois d'un diamètre relativement important et serrées les unes contre les autres, réalisant une figure de morula (fig. 3). Cet aspect en a imposé à plus d'un pour une endosporulation (voir par exemple la figure $7, \mathrm{~B}$, de Redaelli et Cifferi, 1936). Je l'ai rencontré plusieurs fois et, chaque fois, j'ai pu constater que ces sphérules se coloraient intensément par le soudan III et le bleu-nil. Il en est de même pour les cristalloïdes dont j’ai parlé plus haut; ces formations sont dues à une sur-

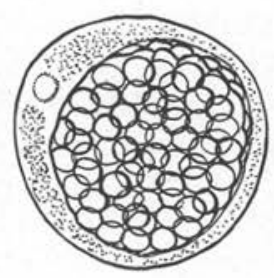

FIG. 3. - Aspect de pseudo - endosporulation (sphẻrules colorables par le soudan III) (souris 362; culture en Ringersérum de 17 jcurs ; à frais). charge excessive de la vacuole en lipides qui détermine une précipitation. Elles n'ont en tout cas absolument rien à voir avec un cycle évolutif du parasite.

Dans la grande majorité des cas, d'ailleurs, le contenu de la vacuole est d'apparence assez homogène. Le rouge neutre la teinte, assez lentement, en jaune-orangé, puis détermine parfois des précipités quand il s'agit d'invidus provenant de cultares en sérum. Le soudan III colore quelques gouttelettes très rares et petites quand il s'agit du milieu ovo-mucoïde ; avec les cultures en sérum les gouttelettes colorées sont bien plus nombreuses et deviennent progressivement coalescentes, si bien qu'en quelques minutes la vacuole entière est colorée (fig. 4). Le bleu de crésyl brillant ne donne absolument aucune métachromasie. Aucune réaction du glycogène ne peut non plus être obtenue ni directement ni après fixation. La vacuole centrale ne contient donc ni métachromatine ni glycogène. Le contenu en est en réalité protido-lipidique, la proportion des lipides étant variable en fonction de la richesse nutritive du milieu et provo- 
quant quand elle est trop forte l'aspect diphasique que nous avons vu plus haut.

$3^{\circ}$ La membrane externe. - Elle est visible sous forme d'un mince liseré hyalin périphérique ; elle a cependant une certaine solidité et elle demande quelque temps pour être traversée par les colorants vitaux ; elle a aussi une élasticité facile à constater quand des courants, dans les préparations en milieu liquide, font déplacer passivement le parasite entre des obstacles ; cette élasticité persiste même après la mort de la cellule ; c'est ainsi qu'en dissociant, dans du liquide de Ringer, le faux-tissu que j'ai dit plus haut avoir observé dans l'intestin de grenouilles, tous les éléments polyédriques, dont
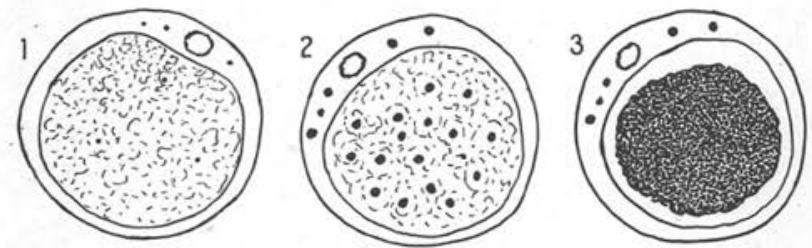

Fıg. 4. - Action du soudan III. 1, individu de culture en ovo-mucoïde : très rares et petites gouttes colorées dans le cytoplasme et la vacuole ; 2,3 , individu de culture en Ringer-sérum, 1 au début, 2 après quelques minutes. (Rana esculenta; culture).

cependant la plupart étaient morts, reprenaient rapidement la forme sphérique. Cependant cette membrane est peu épaisse et la dessiccation la fripe immédiatement, comme on peut facilement le constater sur les éléments placés en bord de lamelle.

Extérieurement à la membrane externe existe souvent un halo composé d'une substance hyaline. Quand il est présent, il est d'épaisseur très variable, allant de moins d'un $\mu$ jusqu'à quatre ou cinq $u$; il n'est visible que par sa réfringence un peu plus forte que celle du parasite et surtout par le fait que les abondantes bactéries du milieu viennent se coller à sa périphérie. Mais ce revêtement manque fréquemment aussi bien chez les individus provenant directement de l'intestin que chez ceux développés en culture et plus souvent encore peut-être chez ces derniers. De plus, quand il existe, il est présent chez de nombreux individus, mais certains cependant en restent dépourvus. Je n'ai pu déterminer à quelle cause est liée la présence ou l'absence de ce halo. Micheletti (1932), qui constate également qu'il peut manquer chez le Blastocystis de l'homme, pense que cela dépend de la fraîcheur des selles et qu'avec le refroidissement il disparait. Je ne puis confirmer cette observation, il peut 
manquer dans des selles humaines très fraîches de même qu'on peut l'observer facilement dans des blastocystes d'animaux à sang froid, ou dans des cultures. Quant à sa nature, it s'agit d'un mucilage et le rouge de ruthénium le colore intensément.

La membrane externe elle-même ne prend pas le bleu-coton et ne présente pas les réactions de la cellulose ; mais le bleu de crésyl brillant la fait apparaître légèrement colorée avec une métachromasie violette ; elle prend de même une teinte rouge-violacée par le bleu de toluidine ; le rouge de ruthénium la colore ; le rouge congo ne donne aucune coloration, contrairement à ce qui se passe pour la membrane des spores.

En dehors des formes sphéroïdes, on peut voir des formes allongées

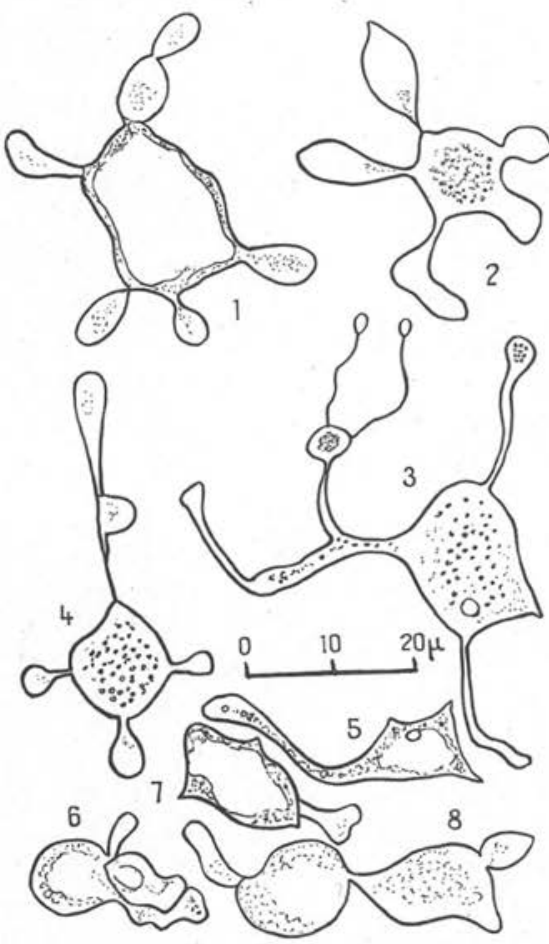

Fig, 5. - Poussée de filaments chez les Blastocystis cultivés en milieu pauvre (aspect à frais) ; $1, z, 3,4$, Rana esculenta 200, ovo-mucoïde, 13 e jour ; 5, Rana temporaria 374, ovo-mucoïde, 15 e jour ; 6, 7, Bufo bufo 371 , ovo-mucoïde, $20^{\mathrm{t}}$ jour ; 8 , Hamopis sanguisuga 203, ovomucoïde, $6^{\circ}$ jour. avec deux noyaux situés chacun à un pôle opposé et présentant un étranglement sensiblement médian. Ces figures avaient été interprétées dès le début comme représentant une division binaire, ce que cependant Redaelli et Ciferri mettent en doute. Il est certain pourtant qu'il s'agit bien d'une division qu'avec un peu de patience on peut suivre au microscope dans les cultures. Outre ce mode de division binaire bien connu, on peut observer surtout dans les cultures des formes irrégulières et bosselées correspondant à une division non plus binaire, mais multiple; de toute façon, il n'y a qu'un petit nombre d'éléments en formation, généralement de taille assez inégale, et la vacuole, si elle présente des amorces d'étranglements multiples, ne modifie du moins en rien son aspect cytologique. Il s'agit d'une division végétative analogue à la précédente, mais où les phénomènes sont accé- 
lérés, la karyotomie étant plus rapide que la plasmotomie. De tels aspects s'observent rarement dans le milieu intestinal, mais couramment dans les cultures ; les bosselures peuvent parfois devenir assez complexes pour réaliser des esquisses de dichotomie ; c'est l'aspect ramifié (verzweigte) de Reyer (1939), que l'on observe dans les cultures au sérum. Mais si l'on s'adresse au milieu pauvre qu'est l'ovo-

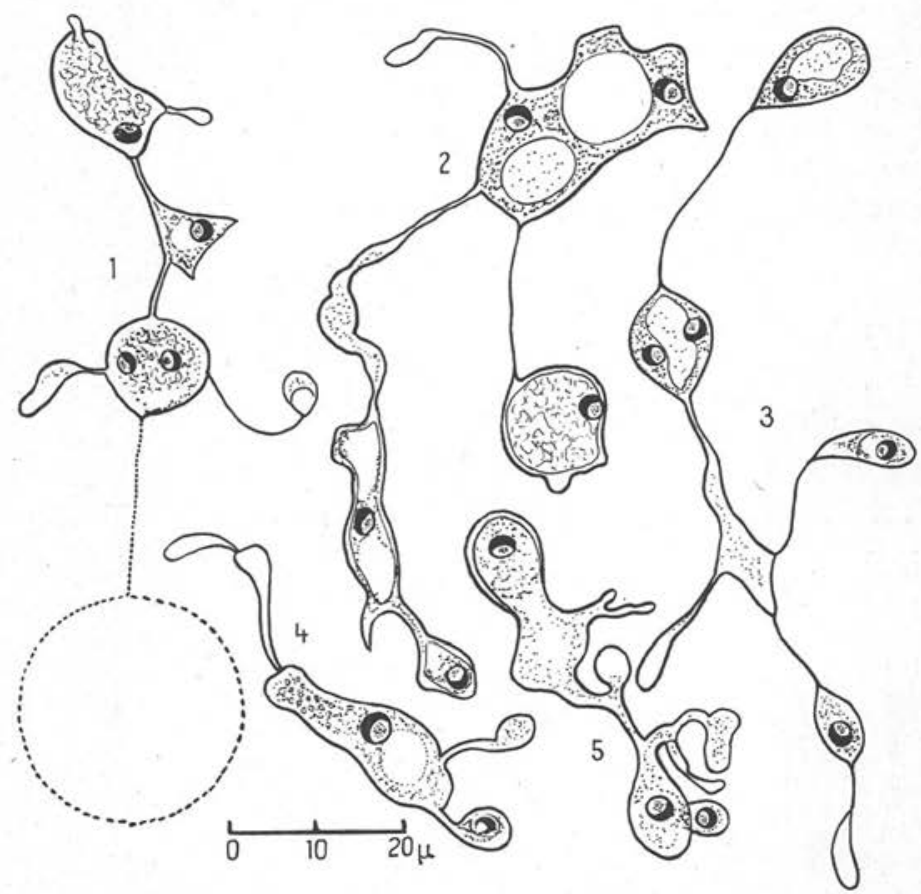

Fic. 6. - Poussée de filaments chez les Blastocys/is cultivés en milieu pauvre (ovo-mucoïde). Aspect après fixation (Bouin) et coloration (hématoxyline ferrique). 1, 2, 3, Rana esculenta $200,15^{\mathrm{e}}$ jour ; 4, 5, Hamopis $203,26^{\circ}$ jour.

mucoïde, on peut observer, dans les cultures de blastocystes d'amphibiens, des individus dont la forme n'est pas sphérique, mais allongée en tubes courts ou même polyédriques et, chez ces derniers, les angles sont souvent le point de départ de processus qui s'élargissent en raquettes ; ces processus filiformes, parfois deviennent fort longs (50 à $60 \mu$ ) et se dilatent par places, formant un chapelet d'élargissements sphéroïdes ; des sortes d'hyphes irrégulières sont ainsi formées ; la paroi en paraît très mince, sauf au niveau des renflements où elle prend l'aspect habituel de celle des Blastocystis (fig. 5). Il ne s'agit pas là d'aspects de dégénérescence, ainsi que l'indiquent les colorants vitaux ; après fixation et coloration, on voit des noyaux d'aspect nor- 
mal, généralement un, rarement deux par rentlement (fig. 6). Ces renflements tiennent assez longtemps entre eux par les filaments qui s'amenuisent progressivement et finissent par se rompre. Chacun des éléments ainsi libérés a, à la forme irrégulière près, la valeur d'un Blastocystis végétatif ; on a ainsi un véritable mode de reproduction par boutures. Avec les blastocystes d'Hæmopis sanguisuga on obtient de même, en ovo-mucoïde, des prolongements tubulaires, plus courts d'aîlleurs qu'avec ceux d'amphibiens, mais présentant aussi des renflements nucléés. Ces aspects sont liés à la pauvreté nutritive du milieu et si, on augmente encore celle-ci, par exemple
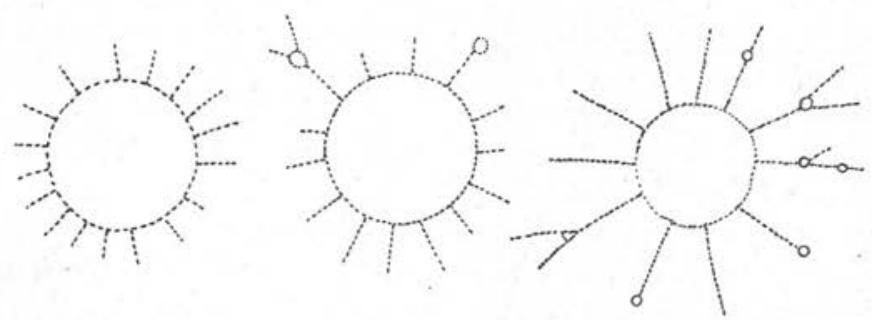

FIG. 7. - Aspeets en oursins obtenus en repiquant en Ringer pur une culture en ovo-mucoïde

en repiquant une culture de Blastocystis en Ringer pur, il n'y a plus multiplication mais les éléments se conservent plusieurs jours et rapidement les sphéroïdes se présentent hérissés de prolongements filiformes dont certains offrent encore des renflements, mais alors de tout petit diamètre et non nucléés (fig. 7). Ainsi se trouve réalisé un curieux aspect en oursin. Il est à noter d'ailleurs que les poussées filamenteuses se font à peu près toujours suivant un nombre variable mais faible de rayons émanant de l'élément primitif, d'où la fréquence des dispositions stellaires.

\section{Sporulation}

La sporulation, signalée tout d'abord par Alexeieff, revue par Beaurepaire-Aragão, Grassé, Schilling et Santoni, s'observe avec la plus grande facilité chez les Blastocystis de toutes provenances en culture au sérum dilué ; elle peut être aisément suivie dans toutes ses phases, du moins à partir du moment où l'attention est attirée sur l'individu qui entre en sporulation.

Il s'agit alors d'un organisme de diamètre variable mais souvent assez important, bien sphérique, avec une pellicule externe très mince et un contenu vacuolaire très clair, d'une réfringence diffé- 
rant peu de celle du milieu de culture ; il a l'aspect d'un ballon bien gonflé ; il est entouré ou non du halo mucilagineux ; il n'y a plus à ce moment de distinction possible entre paroi externe et cytoplasme ; vraisemblablement, tous deux ont fusionné en une couche périphérique commune et ont en plus recu un large apport du contenu de la vacuole qui s'en trouve ainsi clarifiée. C'est dans cette pellicule périphérique, d'abord d'aspect homogène, que l'on voit se dérouler au microscope les phénomènes suivants : des plages de réfringence différente apparaissent ; le nombre en est variable suivant le diamètre de l'individu, mais jamais inférieur à quatre ; elles se remarquent par une densité plus forte et qui va progressivement augmenter ; tout d'abord anguleuses, avec des prolongements à allure pseudopodique, elles se ramassent, prenant provisoirement un aspect losangique ; elles commencent alors à épaissir, à faire saillie sur la sphère qui prend un aspect de boule cloutée. Chacun des îlots ainsi formés est centré par un noyau entouré de grosses mitochondries dans un cytoplasme très riche en glycogène ; à partir du moment où les saillies apparaissent, tout se passe en dix à quinze minutes ; chaque plage continue sa saillie en prenant un aspect arrondi, relié encore à la périphérie par une pédicelle qui s'étire progressivement ; à ce moment, la tête de la saillie est très réfringente par l'abondant matériel mitochondrial qui y est contenu, tandis que le pédicelle a la même apparence terne que ce qui constitue encore la surface externe de l'individu; de la pellicule cytoplasmique de celui-ci il ne reste d'ailleurs à peu près rien qu'un vague fantôme après que les pédicelles se sont rompus et que les spores ont pris la forme sphérique. La presque totalité de ce matériel a donc été utilisée dans la formation des spores. De même, il ne reste du cytoplasme vacuolaire qu'un reliquat très clair, souvent difficile à voir, mais qui conserve encore une certaine viscosité, car il maintient encore en place quelque temps les spores. Quand il existe autour de l'individu qui a sporulé un halo mucilagineux, celui-ci joue aussi un rôle adhésif, mais quand il n'existe pas, les spores suivent bientôt individuellement les moindres courants qui peuvent apparaître dans la préparation et sont rapidement dispersées.

La sporulation, ainsi que je l'ai dit, s'observe sans difficulté dans les cultures en sérum dilué, aussi bien pour le parasite humain que pour ceux d'animaux. Elle apparaît généralement au moment où la culture devient riche, puis avec le temps se raréfie, mais réapparaît dans les subcultures ; en milieu ovo-mucoïde, avec les parasites de poïkilothermes on peut l'observer aussi, mais elle y est plus discrète et plus fugace. Le phénomène se passe-t-il chez l'hôte ? Il n'y a aucun doute en ce qui concerne les poïkilothermes et c'est dans le 
contenu intestinal direct qu'Alexeieff le premier l'avait observé. Je l'ai vu de même chez plusieurs grenouilles (contrairement à Schilling et Santoni) et des sangsues ; il n'y a rien d'impossible à ce qu'il en soit de même chez l'homme, et l'observation courante dans les selles de petits individus à allure de spores est en faveur de cette hypothèse ; il est vraisemblable que si on ne l'a pas encore noté, c'est parce que le phénomène est très rapide, se passe probablement dans la partie la plus haute du côlon et que le péristaltisme d'une part et les obstacles existant dans le bol fécal d'autre part doivent rapidement désagréger les figures de sporulation.

L'aspect des spores (kystes secondaires d'Alexeieff) est bien typique ; elles sont régulièrement sphériques, mesurant en moyenne de 5 à $6 \mu$ de diamètre (peut-être un peu plus chez le parasite d'Hæmopis sanguisuga). La paroi en est relativement épaisse, proportionnellement bien plus que dans les formes végétatives ; le cytoplasme est dense, avec des inclusions très réfringentes, probablement lipidiques ; il n'y a pas encore de vacuole centrale ou alors elle est très petite ; après coloration, le noyau a le même aspect typique que dans les formes végétatives. Le glycogène, si rare dans les formes végétatives, est dans la spore très abondant à l'état diffus ; le soudan III met en évidence quelques gouttelettes lipidiques. La membrane externe présente les mêmes réactions pectiques que celles des individus végétatifs ; elle est en outre colorable par le rouge congo qui ne colore jamais la paroi des adultes. Il n'y a non plus jamais, autour des spores, de halo mucilagineux. Il est vraisemblable que ces éléments, dont la paroi est nettement plus épaisse que celle des adultes et qui doivent donc être plus résistants, sont ceux qui permettent à l'infection de passer d'un individu à un autre.

Mais il n'est pas douteux qu'ils se transforment rapidement en formes végétatives : la vacuole apparaît, augmente de taille, la paroi externe s'amincit, on peut dans les cultures observer ainsi toutes les transitions entre les deux aspects. Dans le cas du parasite de sangsue, cela est particulièrement rapide, au point que l'on voit dans des grappes sporales encore agglomérées par le reliquat structural de la vacuole centrale, les individus encore de petite taille mais ayant déjà tous pris l'aspect végétatif $(9$, fig. 8$)$.

Le nombre des spores formées est variable. Pour les parasites de grenouille, j'ai rencontré une fois le chiffre (minimum) de 4 ; sur 25 processus que j'ai suivis entièrement, j'ai noté trois fois 8 ; une fois 10 ; une fois 11 ; une fois 12 ; deux fois 13 ; deux fois 14 ; cinq fois 16 ; deux fois 18 ; quatre fois 20 ; une fois 27 ; une fois 32 ; une fois 38 ; une fois 39 . Il n'y a done pas de doute que les noyaux se multiplient par division binaire, mais que parfois une ou deux 
divisions se trouvent en avance ou en retard, ce qui explique que l'on n'ait pas rigoureusement des multiples de quatre. Un parasite de provenance humaine m'a montré un nombre de spores variant
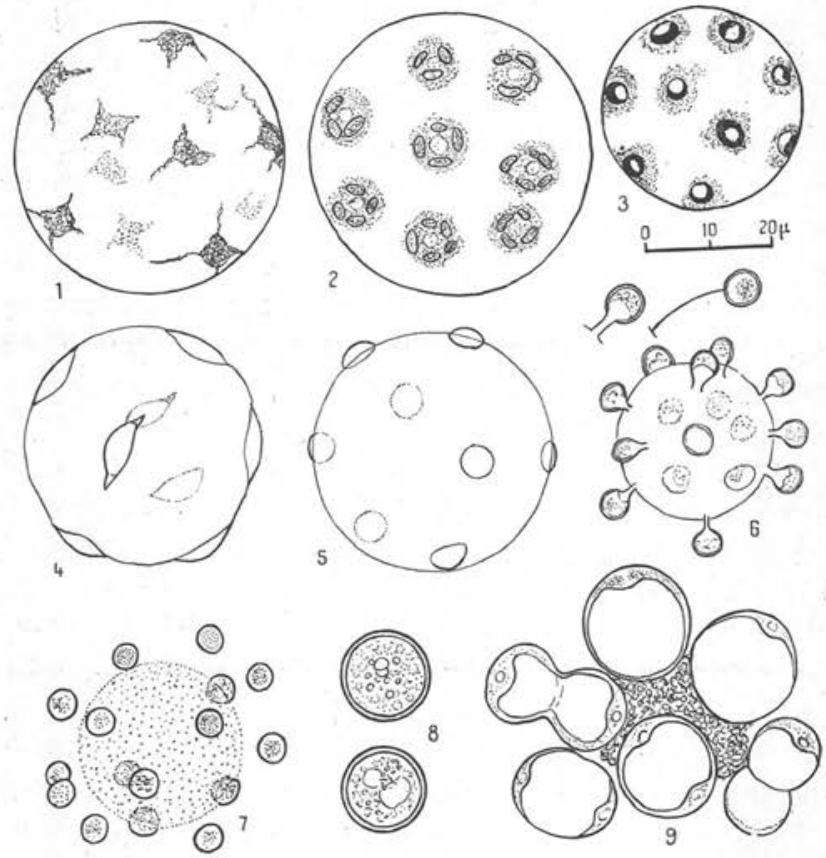

Fig. 8. - Sporulation. 1, formation des plages plus denses de cytoplasme (à frais ; la densité optique de chaque plage a été en réalité exagérée) ; 2, isolement de chaque îlot cytoplasmique avec mitochondries rangées autour du noyau dans un cytoplasme très riche en glycogène (solution iodo-iodurée forte) ; 3 , aspect après fixation et coloration (Bouin-hématoxyline ferrique) ; 4, aspect provisoirement losangique (à frais) ; 5, début de l'arrondissement des spores (à frais) ; 6, formation des exospores (à frais) ; en haut, étirement des pédicelles (à 4 minutes d'intervalle) ; 7 , sporulation terminée, le protoplasme structural de la vacuole primitive ne subsiste qu'à l'état de fantôme ; 8 , deux spores; celle du bas, un peu plus âgée, présente déjà une vacuole (le grossissement ici est bien supérieur à celui des autres figures); 9 , spores restées encore au contact du reliquat de l'individu primitif et ayant déjà pris l'aspect de Blastocystis, dont certains déjà en division. Tout se rapporte au Blastocystis de Rana sauf 2 (Homme) et 9 (Hrmopis).

de 8 à 20. Le parasite d'Hæmopis sanguisuga peut donner des chiffres de même ordre, mais parfois aussi bien plus considérables ; j'ali vu ainsi des amas dépassant la centaine, mais, ainsi que je l'ai signalé plus haut, il y avait déjà des formes en division binaire.

Qu'il y ait là un phénomène de sporulation ne saurait faire le moindre doute, mais il est tout aussi certain, contrairement à ce 
qu'ont écrit la majorité des auteurs (Schilling et Santoni exceptés), qu'il n'y a pas endosporulation. Il suffit de suivre le phénomène pour se rendre compte que les spores sont externes, formées à la surface et même aux dépens de la partie superficielle, qui s'en trouve ainsi résorbée ; les spores ne sont contenues dans aucune enveloppe, à moins que l'ont n'ait pris pour telle la couche mucilagineuse dont la présence est d'ailleúrs loin d'être constante. Il n'y a en réalité, chez les Blastocystis, aucune endosporulation, et ce qui a été allégué à ce sujet ne vient que de mauvaises interprétations.

On peut enfin se demander, connaissant la façon dont se fait la sporulation, si les formations hyphoïdes curieuses, à aspect si fréquemment stellaire et les aspects hérissés en oursins que j'ai décrits plus haut et qui apparaissent en milieux pauvres, ne correspondent pas à une exosporulation qui s'amorcerait et se déroulerait de façon atypique et très fragmentaire par suite des conditions nutritives.

Alexeieff, en décrivant la sporulation qu'il appelait formation des kystes secondaires, avait admis que le phénomène était précédé d'une autogamie par fusion dans un individu de deux noyaux en un seul qui par divisions rapides donne les noyaux sporaux ; peu après il admettait par contre la fusion des noyaux de deux individus se comportant comme gamètes. Je n'ai rien vu de semblable ; je ne saurais cependant nier la possibilité, au début de la sporogonie, d'un processus quelconque intéressant le noyau, car les tout premiers stades du phénomène sont particulièrement difficiles à dépister, et seules de nouvelles recherches pourraient élucider ce point.

$$
* *
$$

De ce qui précède, que peut-on déduire au point de vue du statut des Blastocystis ?

Tout d'abord une chose est certaine, c'est leur nature végétale. Certes, on a reconnu depuis longtemps qu'il ne s'agit pas de kystes de Trichomonas, ni d'autres flagellés ; bien qu'à un moment Chatton ait paru emporter cette conviction avec des parasites de Tarentola mauritanica, Grassé a montré qu'il avait pris pour des Blastocystis des kystoïdes de Prowazekella lacertæ. Cependant il y a eu encore, par la suite, quelques défenseurs attardés de cette thèse ; Diego Guevara Pozo expose encore en 1935 que les Blastocystis ne constituent pas une entité propre, mais seulement une forme que prennent des flagellés intestinaux pour se défendre contre la diminution de pression osmotique du milieu ; c'est ce qu'il nomme blastocystisation des flagellés. Cette théorie est entièrement insoutenable devant les résultats que donnent les cultures aussi bien des flagellés intes- 
tinaux que de blastocystes : l'arrondissement et la vacuolisation des flagellés intestinaux sont des phénomènes de dégénérescence qui n'ont absolument rien à voir avec les Blastocystis.

Mais où les classer parmi les végétaux ? Alexeieff, suivi par beaucoup d'auteurs, les considérait dès 1910 comme des levures. Redaelli et Ciferri par contre ont émis en 1935 et développé en 1926 l'idée, assez inattendue, qu'il s'agit d'une algue incolore, parce que : $1^{\circ}$ le corps central peut être assimilé à un chromatophore (opinion en réalité absolument insoutenable au point de vue cytologique) ; $2^{\circ}$ la capsule mucilagineuse externe est rare chez les levures ; $3^{\circ}$ la formation des «endospores 》 ne rappelle pas ce qu'on voit dans les levures (mais nous avons vu qu'il n'y a pas d'endospores) ; $4^{\circ}$ les kystoïdes de Blastocystis peuvent atteindre de grandes tailles mais ne sont pas alors comparables aux formes géantes stériles qu'on observe chez les levures ; $5^{\circ}$ la division nucléaire se fait par amitose dans les algues vertes (mais la division du noyau de Blastocystis qui doit d'ailleurs être très rapide n'a pas été suivie en détail) ; $6^{\circ}$ quelques chlorophycées ont été rencontrées comme parasites intestinaux $; 7^{\circ}$ le seul mode de multiplication est la formation d'endospores (R. et $\mathrm{C}$. nient en effet la division végétative simple ; ils préfèrent voir dans les aspects bien connus de cette division des stades de dégénérescence). On voit l'extrême faiblesse de ces arguments, et aussi bien cette thèse n'a pas été admise par les auteurs suivants (Reyer, Schilling et Santoni). Elle est en réalité inadmissible.

Mais on n'a pas le droit pour autant de ranger les Blastocystis dans les levüres du moins les levures ascosporées, comme le faisait Alexeieff. Rien ne permet ce rapprochement, ni le mode de formation des spores, ni la cytologie nettement différente (1).

Alexeieff d'autre part réunissait aux Blastocystis les Dermocystidium en une famille, celle des Blastocystidées. Les Dermocystidium sont des organismes formant dans la peau d'amphibiens et sur les branchies de poissons de petites tumeurs contenant des masses de petites spores arrondies et ayant à leur intérieur une inclusion sphérique réfringente; mais c'est à cela que se borne la ressemblance ; ces spores sont contenues dans un sporange avec paroi externe et cloisonnement intérieur ; elles y sont formées après un stade plasmodial ; enfin P. de Beauchamp a montré qu'à maturité ces spores sont des zoospores uniflagellés. Il s'agit donc d'une incontestable Chytridiale.

(1) A. Guilliermond, qui avait vérifié mon étude cytologique de Blastocystis (1937), m'avait dit n'avoir vu en eux aucun caractère cytologique comparable à ceux des levures. 
En réalité, la structure vésiculaire des Blastocystis, leur mode de sporulation externe avec pédicelles périphériques, évoque deux rapprochements : vers Paracoccidiö̈des brasiliensis d'une part, vers Boudierella coronata d'autre part.

Paracoccidioïdes brasiliensis (Splendore, 1912) donne une blastomycose cutanéo-muqueuse, dans les lésions de laquelle le cham-

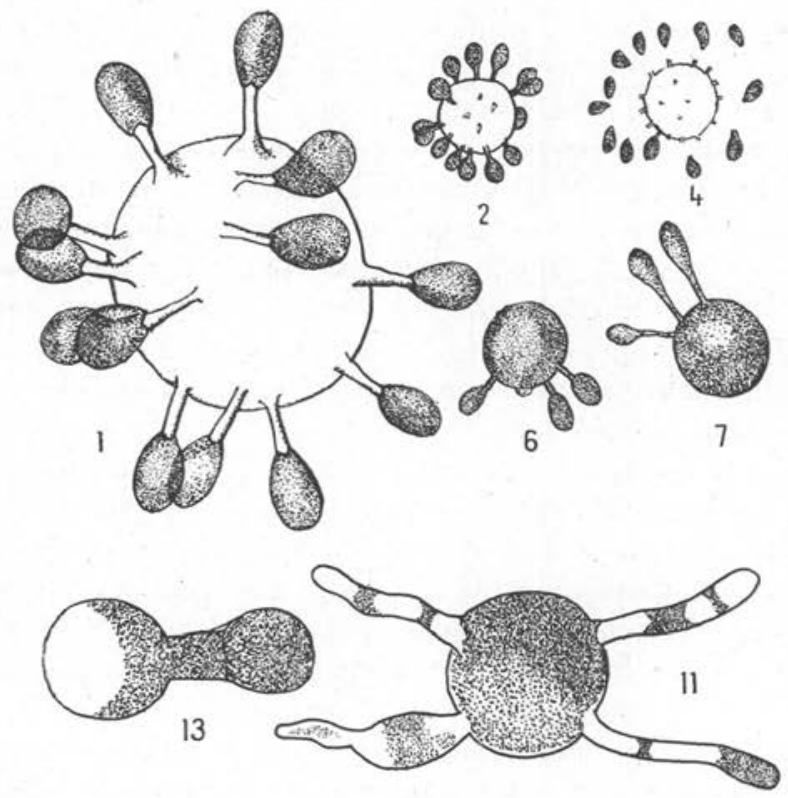

FIG. 9. - Bondierella coronata. Reproduction des fig. 2, 3, 4, 6, 7, pl. IV et 11, 13 , pl. V de Costantin (les numéros ont été conservés). Les légendes originales! en sont : pl. IV : 2, spore ayant germé et produit un certain nombre de conidies secondaires ; 3 , mème stade, dessin plus fortement grossi ; 4, spore ayant projeté les conidies secondaires ; 6 et 7 , germinations de spores; pl. V : 11, spore donnant, au lieu de stérigmates, des tube, germinatifs; 13, spore donnant naissance à une spore seendaire identique à elle. [Pas de grossissement indiqué].

pignon se présente avec l'aspect d'une masse sphérique réfringente de 25 à $30 \mu$ de diamètre, entourée de petites masses arrondies de $1 \mu$ environ; dans les cultures on obtient des masses arrondies analogues à celles des lésions, et, dans certains milieuxx, des filaments mais sans conidies. La position systématique des Coccidiö̈des et Paracoccidioïdes est discutée. O. da Fonseca et Leão les rattachent aux Hémiascomycètes ; tandis que M. Langeron soutient depuis longtemps un rapprochement avec les Archimycètes et les Phy-

Ans. de Parasitologie, T. XXVII, $\mathrm{N}^{\circ} 1-2-3 .-1952$. 
comycètes et signale (1945), en ce qui concerne Paracoccidioüdes, l'analogie de sporulation avec Boudierella.

Boudierella coronata a été décrite en 1897 par J. Costantin ; il s'agit d'un champignon apparu spontanément dans les cultures de germination de champignon de couche, et saprophyte, mais probablement non parasité ; il se présente sous forme de sphères (spores) développant à leur périphérie 18 à 16 conidies secondaires portées sur des longs pédicelles ; tout le cytoplasme de la spore passe dans les spores secondaires ; il y a parfois des irrégularités de sporulation, les spores en petit nombre n'apparaissant alors que sur une partie de la sphère. Parfois, au lieu de donner des stérigmates, les éléments donnent des tubes courts et larges ou même s'étranglent pour donner des spores secondaires. Il y a une analogie très étroite entre les aspects des Blastocystis que j'ai rapportés plus haut et ceux que représente Costantin (fig. 9). Bien que cet auteur n'ait pas observé de phénomènes de sexualité, il range Boudierella, qui ne paraît pas avoir été revue depuis, parmi les Entomophtoracées (Phycomycètes, Zygomycètes).

Notons de plus qu'on rencontre de façon courante, parmi les amphibiens, des éléments sphéroïdes et à grosses vacuoles, ayant alors une allure qui rappelle fort les Blastocystis, mais parfois plus allongés (1) et dans tous les cas avec une paroi nettement plus épaisse et un cytoplasme périphérique plus dense. Il s'agit de Basidiobolus ranarum incontestable Entomophtorale connue de longue date et dont le cycle évolutif a été décrit par Levisohn (1927). Dans les milieux de culture pour Blastocystis, ces éléments ne se modifient pas, mais sur milieux solides ils germent facilement donnant un thalle typique.

En définitive, les caractères morphologiques et cytologiques des Blastocystis permettent de les placer, non dans les levures, mais à côté de champignons qui, encore que leur statut ne soit pas entièrement certain, peuvent être rangés dans les Phycomycètes et parmi ceux-ci dans les Entomophtorales, ou à leur voisinage immédiat. Et cela donne un intérêt spécial à la recherche de phénomènes de sexualité que je n'ai point observés mais qui ne sont pas impossibles.

\section{RÉSUMÉ}

Les Blastocystis mis en culture dans ces milieux pauvres (ovomucoïde) présentent des poussées filamenteuses en raquettes, parmi

(1) C'est ainsi que la fig. 121, de la pl. XII de Grassé (1926), dont la légende est : «Développement (?) d'une spore [de Blastocystis de Bufo vulgaris] 》 représente un élément de Basidiobolus ranarum. 
lesquelles se différencient des renflements nucléés qui après séparation peuvent évoluer pour leur compte ; il se réalise ainsi un mode de dissémination par bouture. L'aspect stellaire de ces poussées permet de penser qu'il n'y a là que l'altération du processus normal de sporulation.

La sporulation est observable chez les Blastocystis de toutes provenances dans les cultures en milieu convenable. Il n'y a jamais formation d'endospores, mais une exosporulation où les spores périphériques sont formés en épuisant l'ensemble de l'individu qui sporule. Ces spores sont probablement des formes plus résistantes que les formes ordinaires issues de division végétative, et comme telles servent peut-être à la propagation parasitaire d'individu à individu. Mais elles prennent rapidement l'aspect des formes ordinaires.

Ce mode bien typique de sporulation permet de rapprocher les Blastocystis d'une part des Paracoccidioüdes et d'autre part de Boudierella, Entomophtorée qui présente avec eux des aspects d'une similitude frappante. Les Blastocystis peuvent donc être rangés dans les Phycomycètes et de façon plus précise parmi les Entomophtorales ou à leur voisinage immédiat.

\section{BIBLIOGRAPHIE}

Alexerefr (A.). - Sur les \& kystes de Trichomonas intestinalis » dans l'intesỉin des Batraciers. Bull. Scient. Fr. ot Belgiqne, XLIV, 1910, p. 33.

Sur la nature des formations dites « kystes de Trichomonas ». C.R. Soc. de Biol., LXXI, 1911, p. 296.

Sur quelques protistes parasites intestinaux d'une Tortue de Ceylan (Nicoria trijuga), Zool. Anz., XL, 1912, p. 97.

- Notes protistologiques... $3^{\circ} \mathrm{A}$ propos de la faune intestiıale d'Hamopis sanguisuga. Zool. Anz., XI.III, 1914, p. 521.

BACH (F. W.) et Krefer (K. H).-- Untersuchungen über Blastocystis. Centralbl. f. Bakl, I Abt, Or., LXXXıX, 1922, p. 72.

Barret (H. P.). - A method for the cultivation of Blastocystis. Ann. Trop. Mcd. and Paras., XV, 1921, p. 113.

Beaurepaire-Aragao (H. de). - Estudos sobre os Blastocystis. Mem. dos Inst. Osw. Cruz, 1922, p. 241.

Brumpt (E.). - Colite à Tetramitus mesnili (Wenyon 1910) et colite à Trichomonas hominis Leuckart, 1879. Blastocystis hominis n. sp. et formes voisines. Bull. Soc. Path. exot., V, 1912, p. 725.

-. Précis de Parasitologie, Paris, Masson, 6e éd., 1949, II, p. 2006.

Costantin (J.). - Sur une Entomophtorée nouvelle. Bull. Soc. Mycol. de France, XIII, 1897 , p. : $: 8$.

Grassé (P.-P.). - Contribution à l'étude des Flagellés Parasites. Arch. Zool. exp. et gén., LXV, 1926, p. 345.

Guevara Pozo (D.). - Blastocystizacion de los flagelados. Bol. Univers. de Grenada, VII, 1935 , p. 391. 
Langeron (M.). - Précis de Mycologie, Paris, Masson, 1945, p. 646.

LAviER (G.). - Sur certaines formes que présentent en culture les Blastocystis. C.R. Soc. de Biol., CXXV, 1937, p. 593.

- Sur la cytologie des protistes du genre Blastocystis. C.R. Ac. des Sc., CCV, 1937, p. 340 .

Lxich (K. M.). - Blastocystis species in culture. Amer. J. of. trop. Med., II, 1922 , p. 215.

Michelettr (E.). - Contributo allo studio del \& Blastocystis jalinus » (Perroncito). Ann. di Med. nav. e colon., XXXVIII, 1932, vol. 1, p. 9.

- Nuovo contributo allo studio del \&Blastocystis jalinus 》 (Perroncito). Ibidem, XXXIX, 1933, vol. 1 , p. 159 .

Redaelir (P.) et Ciffrui (P.). - Una nuova ipotesi sulla natura di \& Blastoeystis hominis x. Boll. Soc. Ital. Biol. sper., X, 1935, p. 816.

- Argomenti a favore di una sistemazione del genere \& Blastocystis » nelle Algae. Boll. Istit. Sieroterap. Milanese, XV, 1936, p. 154.

Reyer (W.). - Ueber den Sitz von Blastozystis in Derm. Zentralbl. f. Bakt., I Abt., Or., CXLII, 19:38, p. 323.

- Ueber die Vermehrung von Blastocystis in der Kultur. Arch. f. Protistenk., XCII, 1939, p. 226.

Schilling (C.) et Santoni (D. A.). - Ueber Blastozystis. Zentralbl. f. Bakt., I Abt., Or., XCCCrII, 1936, p. 293.

Wenyon (C. M.). - Observations on the intestinal Protozoa of three egyptian Lizards, with a note on a cell-invading fungus. Parasitology, XII, 1920 , p. 350 .

Wenyon (C. M.) et O'Connor (F. W.). - Human intestinal Protozoa in the Near-East, Londres, John Bale and Danielsson, 1917, p. 84. 